\title{
Community assembly processes as a mechanistic explanation of the predator-prey diversity relationship in marine microbes
}

\author{
Feng-Hsun Chang ${ }^{1}$, Jinny Yang ${ }^{2}$, Ariana Chih-Hsien Liu ${ }^{1}$, Hsiao-Pei Lu ${ }^{3}$, Gwo-Ching \\ Gong $^{4}$, Fuh-Kwo Shiah ${ }^{5}$, and Chih-hao Hsieh ${ }^{1}$ \\ ${ }^{1}$ National Taiwan University \\ ${ }^{2}$ University of Michigan Department of Ecology and Evolutionary Biology \\ ${ }^{3}$ National Cheng Kung University \\ ${ }^{4}$ National Taiwan Ocean University \\ ${ }^{5}$ Academia Sinica
}

November 6, 2020

\begin{abstract}
Predator and prey $\alpha$-diversity are often positively associated; yet, underlying mechanisms remain unclear. We attempt to address this issue by deciphering how $\alpha$-diversity of predator and prey influences each other's community assembly processes and subsequently determines $\alpha$-diversity. The occurrence of assembly processes were indicated by the mean pairwise taxonomic index within a community ( $а M \Pi T I)$, assuming assembly processes left traceable imprints on species' phylogeny. Specifically, $a M \Pi T I$ quantifies deviations of observed phylogenetic distances from that of random, thus indicating that non-random/deterministic assembly processes are in action. Less negative $a M \Pi T I$, which hints at the occurrence of weaker homogeneous deterministic assembly processes, is expected to increase $\alpha$-diversity of the community. We hypothesize that higher predator and prey $\alpha$ diversity make each other's aMПTI less negative, which then increases their $\alpha$-diversity. To test the hypothesis, we calculated Shannon diversity and $а M \Pi T I$ for heterotrophic nanoflagellates (HNF; predator) and bacteria (prey) communities in the East China Sea. The HNF Shannon diversity was found to make the aMПTI of bacteria less negative, which then increased bacterial Shannon diversity. In contrast, bacterial Shannon diversity did not affect HNF's aMПTI. We provide evidence that top-down control underpins the positive $\alpha$-diversity association among trophic levels in microbes of the East China Sea.
\end{abstract}

\section{Hosted file}

PdPy_ADiv_Assemb.pdf available at https://authorea.com/users/373560/articles/491224community-assembly-processes-as-a-mechanistic-explanation-of-the-predator-prey-

diversity-relationship-in-marine-microbes 